\title{
Efavirenz Conceptions and Regimen Management in a Prospective Cohort of Women on Antiretroviral Therapy
}

\author{
Sheree Schwartz, ${ }^{1,2}$ Taha E. Taha, ${ }^{2}$ Willem Daniel Francois Venter, ${ }^{1}$ Shruti Mehta, ${ }^{2}$ \\ Helen Rees, ${ }^{1}$ and Vivian Black ${ }^{1}$ \\ ${ }^{1}$ Wits Reproductive Health and HIV Institute, University of the Witwatersrand, 27516 Johannesburg, South Africa \\ ${ }^{2}$ Department of Epidemiology, Johns Hopkins Bloomberg School of Public Health, Baltimore, 21205 MD, USA
}

Correspondence should be addressed to Sheree Schwartz, sschwartz@wrhi.co.za

Received 19 January 2012; Revised 17 April 2012; Accepted 18 April 2012

Academic Editor: Susan Cu-Uvin

Copyright (C) 2012 Sheree Schwartz et al. This is an open access article distributed under the Creative Commons Attribution License, which permits unrestricted use, distribution, and reproduction in any medium, provided the original work is properly cited.

\begin{abstract}
Use of the antiretroviral drug efavirenz (EFV) is not recommended by the WHO or South African HIV treatment guidelines during the first trimester of pregnancy due to potential fetal teratogenicity; there is little evidence of how clinicians manage EFV-related fertility concerns. Women on antiretroviral therapy (ART) were enrolled into a prospective cohort in four public clinics in Johannesburg, South Africa. Fertility intentions, ART regimens, and pregnancy testing were routinely assessed during visits. Women reporting that they were trying to conceive while on EFV were referred for regimen changes. Kaplan-Meier estimators were used to assess incidence across ART regimens. From the 822 women with followup visits between August 2009-March 2011, 170 pregnancies were detected during study followup, including 56 EFV conceptions. Pregnancy incidence rates were comparable across $\mathrm{EFV}$, nevirapine, and lopinavir/ritonavir person-years $(95 \% 100 /$ users $(P=0.25))$; incidence rates on EFV were $18.6 \mathrm{Confidence}$ Interval: 14.2-24.2). Treatment substitution from EFV was made for 57 women, due to pregnancy intentions or actual pregnancy; however, regimen changes were not systematically applied across women. High rates of pregnancy on EFV and inconsistencies in treatment management suggest that clearer guidelines are needed regarding how to manage fertility-related issues in. women on EFV-based regimens.
\end{abstract}

\section{Introduction}

Although HIV reduces fertility, an increase in pregnancy incidence has been documented in HIV-infected women using antiretroviral therapy (ART) [1-3]. Combination firstline regimens in Sub-Saharan Africa typically are comprised of two nucleoside/nucleotide reverse transcriptase inhibitors (NRTIs) and one nonnucleoside reverse transcriptase inhibitor (NNRTI). The NNRTI drugs commonly used in the region are nevirapine (NVP) and efavirenz (EFV); the drugs have comparable clinical performance, but different toxicity profiles [4]. Due to concerns over possible EFV teratogenicity, treatment management for women must account for reproductive potential in addition to drug interactions and toxicities.

Efavirenz-related pregnancy concerns are largely based on a study in cynomolgus monkeys in which anencephaly, a neural tube defect (NTD), was linked to EFV exposure during pregnancy; a case of microphthalmia and one case of cleft palate were also observed in the monkeys exposed to EFV [5]. Six retrospective cases and one prospective NTD have been reported in human infants exposed to EFV during pregnancy [6]. Based on this evidence, EFV is considered potentially teratogenic and is contra-indicated for the first trimester of pregnancy when NTDs occur. Systematic reviews, however, have found no association between EFV exposure and birth defects [7-9]; recent reports from West Africa and South Africa similarly found no evidence of EFV-related teratogenicity $[10,11]$. Despite these assurances, concerns remain and the most recent adult HIV treatment guidelines from the World Health Organization (WHO) and the South African Department of Health, which has the largest ART treatment program in the world, counsel against first trimester EFV exposure $[12,13]$. 
The frequency of EFV conceptions is largely unknown. Most pregnancy-related data on EFV conceptions is reported from pregnancy registries and retrospective file review, from which incidence estimates are typically underestimated as pregnancies not carried to term due to spontaneous abortion or elective termination are frequently not captured through registry and file review. Information on how providers manage fertility-related issues in women on EFV is also limited. WHO guidelines recommend substituting EFV with either NVP or a protease inhibitor (PI) if the pregnancy is $\leq 28$ days gestation [12]. South African National Treatment Guidelines recommend substituting EFV for NVP in the first 12 weeks of pregnancy; the guidelines do not refer to $\mathrm{CD} 4$ cell count in relation to drug choice for regimen substitution. How closely these guidelines are followed is unknown. Neither WHO nor South African guidelines provide direction for regimen changes amongst women trying to conceive who are already using EFV.

The objectives of this study are to prospectively compare pregnancy rates by ART regimens in an operational setting and to assess HIV treatment management of fertility-related issues amongst women on EFV-based regimens.

\section{Methods}

2.1. Cohort Description. Women on ART or being initiated onto ART were enrolled for prospective followup in four public-run HIV clinics in Johannesburg, South Africa, from August 2009 to January 2010. A t the time of study enrollment, lifelong ART was freely available for adults in South Africa with CD 4 counts $<200$ cells $/ \mu \mathrm{L}$ (this was universally increased to 350 cells $/ \mu \mathrm{L}$ in August 2011, well after study enrollment was complete). First-line regimens include EFV or NVP and 2 NRTIs; second-line therapy is typically comprised of 2 NRTIs and a PI (lopinavir/ritonavir). The study examined fertility-related outcomes in women on ART and has been previously described [14]. Briefly, 850 women were enrolled and followed for incident pregnancy for one year; women who conceived during study followup were followed throughout the duration of their pregnancies in order to obtain pregnancy outcomes. Followup for the primary pregnancy incidence endpoint was completed in March 2011; followup of pregnancy outcomes was completed in December 2011. Women were eligible for enrollment if they were aged 18-35 years, on ART, not pregnant, not breastfeeding, sexually active in the past year, and had not been previously sterilized. Pregnancy testing was conducted at enrollment to exclude prevalent pregnancies.

2.2. Assessment of Exposure and Outcome Variables. Structured interviews covering an array of questions on demographics, health, and reproductive histories were conducted at study enrollment. Fertility intentions, contraceptive use, and urine-based pregnancy testing were prospectively measured by study staff during participants' routine clinic visits. As this was a clinical cohort established within an operational care setting, visit schedules were determined by providers; on average, participants were seen every two months.
At baseline, women were asked about their current and future fertility plans. During followup, fertility intentions were assessed at each visit by asking participants if they were currently trying to get pregnant. Pregnancy was assessed at each visit through urine-based testing for human chorionic gonadotropin (hCG); repeat urine testing was immediately performed for all positive pregnancy tests. Pregnancy was defined as having two same-day positive pregnancy tests. Conception dates were assigned to two weeks following the last menstrual period or if unknown, to 266 days before the due date determined by ultrasound and recorded in antenatal records. Ultrasound was done free of charge at the discretion of participants' health providers. Pregnancies occurring during the study were followed through pregnancy duration.

ART treatment and clinical data were confirmed through pharmacy records, medical chart review, and laboratory records. Due to EFV-related teratogenicity concerns, all women conceiving or trying to conceive on EFV during the study were referred for a regimen change. Women conceiving on EFV were counseled about potential risks, reassured that termination was not required because of the EFV conception, and referred for free fetal abnormality scans at a tertiary clinic.

The study was approved by the University of the Witwatersrand Human Research Ethics Committee, Johannesburg, South Africa; all participants provided written informed consent.

2.3. Statistical Analysis. Survival analysis was used to assess time-to-pregnancy by ART regimen. The origin for the survival analyses was study enrollment, and the outcome of interest was incident pregnancy. Women were censored when they experienced the outcome, died, were lost-to-followup or completed the study. For women with multiple pregnancies during study followup, only the first pregnancy was included in the survival analyses. Kaplan-Meier estimators were used to assess one-year cumulative incidence for pregnancy. Equality of failure functions was assessed through logrank analysis. Both a "per-protocol" and a "per routine care" analyses were conducted to assess the impact of the study on EFV-related conceptions. In the per-protocol analysis, ART regimens were treated as time-varying; ART treatment was lagged one visit to ensure that treatment exposure reflected participants' regimens at time of conception. Per study protocol, women trying to conceive on EFV were referred for regimen changes due to safety concerns. In the absence of this study intervention (patient referrals), regimen changes would have been unlikely, as fertility intentions are not routinely assessed by providers after ART initiation and no consistent policies are in place to change women stable on EFV to other regimens if they are trying to conceive. The per routine care analysis assessed one-year pregnancy incidence according to participant regimens at time of study enrollment; this likely would have been the counterfactual regimen at time-of-conception had the research study not intervened.

Pregnancy incidence by ART regimen according to time on ART was also assessed using Kaplan-Meier estimators. 


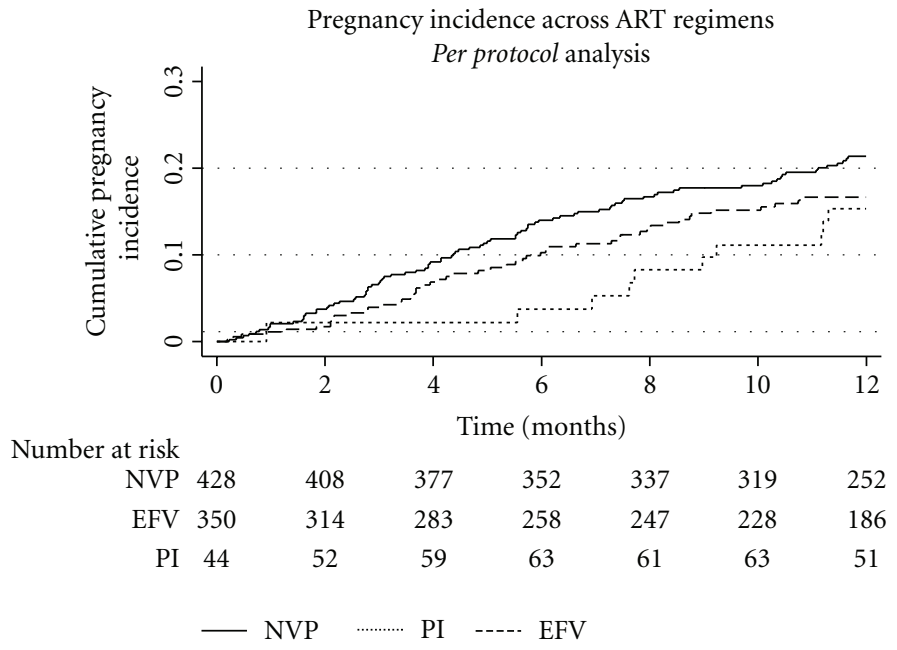

(a)

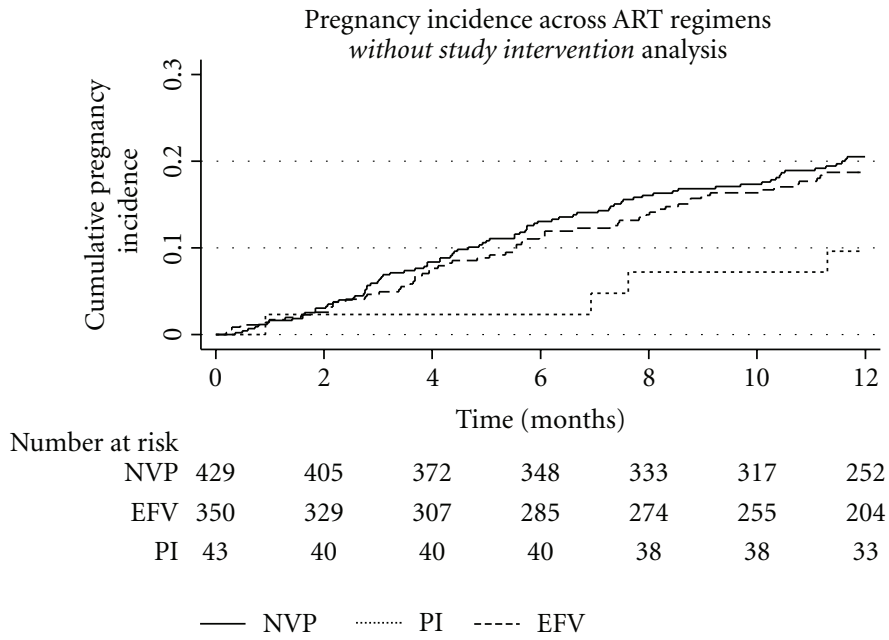

(b)

FIgURE 1: Pregnancy incidence across antiretroviral therapy (ART) regimens according to (a) treatment at time of conception and (b) treatment had the study not intervened to refer participants for regimen change. NVP: Nevirapine; EFV: Efavirenz; PI: Protease Inhibitor (lopinavir/ritonavir).

The outcome of interest for this analysis was incident pregnancy, and the key exposure was time-varying ART. The time origin for this analysis was ART initiation; the analysis thus allowed for individuals in the study to have late entries and early exits from the analysis according to their time on ART. Censoring, multiple pregnancies, and comparisons were handled as described above. Comparisons of medians and proportions between groups were assessed using Wilcoxon rank-sum tests and chi-square statistics.

\section{Results}

Of the 850 women enrolled into the study, 822 (97\%) had at least one followup visit between August 2009 and March 2011 and 734 (86\%) completed study followup. Participants contributing to followup had a median of 6 visits over 12 months. The median time-on-ART at enrollment into the study was 13 months (interquartile range [IQR]: 5-24), and the median CD4 count was 320 cells/ $\mu \mathrm{L}$ (IQR: 178-473). At enrollment, 429 women were on NVP, 350 on EFV and 43 on $\mathrm{LPV} / \mathrm{r}$-based regimens.

Overall 170 incident pregnancies were detected in 161 women ( 8 women were pregnant $>1$ time). Women contributed a total of 745 person-years (PY) at-risk for pregnancy; only time-at-risk for first pregnancy during study followup was included in the incidence analysis. Pregnancy incidence rates by regimen were 24.3/100 PY (95\% Confidence Interval [CI]: 19.9-29.7) on NVP, 18.6/100 PY (95\% CI: 14.2-24.2) on EFV and 18.8/100 PY (95\% CI: 10.7-33.1) on LPV/r. Kaplan-Meier curves (Figure 1) demonstrate the one-year cumulative incidence of pregnancy across treatment arms. Incidence rates in the per-protocol analysis were slightly higher in women on NVP as compared to EFV throughout study followup (Figure 1(a)), although rates 
were not statistically significantly different between the two groups (log-rank NVP versus EFV: $P=0.11$ ). Because the research study intervened with patient care to refer women trying to conceive on EFV for regimen changes, the perroutine care analysis (Figure 1(b)) illustrates pregnancy rates by regimen at study enrollment. In this analysis, differences in pregnancy incidence between the NVP and EFV arms were minimal (log-rank NVP versus EFV: $P=0.48$ ).

Kaplan-Meier curves in Figure 2 assess the cumulative incidence of pregnancy on ART regimens during the 12 months of study followup, according to time on ART. Incidence across ART regimens was not statistically significantly different between EFV and NVP users (log-rank $P=0.09$ ) and illustrates similar inferences to the previous analyses. Figure 2 also demonstrates, however, that the cumulative burden of pregnancy on EFV will be high over time, as women of reproductive age continue treatment on this regimen. In this analysis, $40 \%$ of women on EFV who had already been on ART for 3 years upon study entry, conceived on EFV during followup.

Details of fetal exposure to EFV and fertility-related ART regimen substitutions are presented in Table 1. In total, 56 EFV conceptions were detected in 54 women. Twenty-five women with EFV conceptions received regimen changes during pregnancy; average time to regimen change amongst those actually changed was 6 weeks after conception [IQR: 4-8]. Nine pregnant women continued on EFV due to late detection $(n=4)$, clinic delays $(n=1)$, and indecision over pregnancy termination $(n=4)$. Thirty-four percent of EFV conceptions were terminated prior to regimen changes.

Amongst sexually active women not trying to conceive, hormonal contraceptive use over followup was $32.9 \%$ in women on NVP, $25.7 \%$ on EFV, and $37.5 \%$ on LPV/r $(P<$ $0.01)$. Hormonal contraceptive use amongst women not trying to conceive was also lower in EFV versus non-EFV users at enrollment (EFV 28\% versus non-EFV 35\%, $P=0.06$ ). One EFV conception was attributed to an injectable contraceptive failure. Pregnancy incidence rates amongst hormonal contraception users on EFV versus NVP were 1.5/100 PY [95\% CI: 0.2-10.9] and 7.1/100 PY [95\% CI: 3.4-14.9], respectively; contraceptive failures were too infrequent and confidence intervals too wide for meaningful comparisons across hormonal contraceptive methods.

During followup, 96/350 (27\%) women on EFV at enrollment had at least one visit in which they were trying to conceive on EFV, including fifteen women with planned pregnancies while on EFV-based regimens. All women trying to conceive on EFV were referred for regimen changes; however, records and patient reports suggested many providers would only substitute regimens after an established pregnancy to avoid unnecessary treatment changes. Thirty-two women received preventive regimen changes from EFV due to their fertility intentions; of these, 6 (19\%) became pregnant during followup.

A total of 57 fertility-related regimen changes were made during the study due to either EFV conceptions or intention to conceive while on EFV. NVP was substituted for EFV 67\% of the time and LPV/r 33\% of the time. Women switched to LPV/r versus NVP had higher median CD4 counts (573

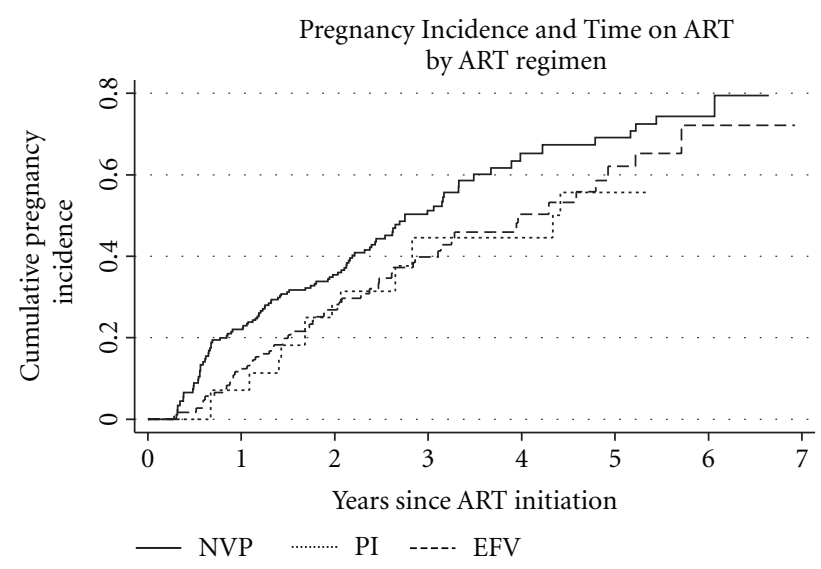

FIGURE 2: Cumulative incidence of pregnancy during study followup by years on antiretroviral therapy (ART); regimen arms represent time-varying treatment exposure during study followup. NVP: nevirapine-based regimen; EFV: efavirenz-based regimen; PI: boosted-protease inhibitor-(lopinavir/ritonavir) based regimen.

versus 257 cells $/ \mu \mathrm{L}, P<0.01)$. Median viral load and acute liver damage (measured through alanine aminotransferase) were equivalent across groups $(P=0.76$ and $P=0.80)$.

Pregnancy outcomes for the $56 \mathrm{EFV}$ conceptions were: live birth $(n=26)$, termination of pregnancy $(n=19)$, miscarriage $(n=9)$, and loss to followup $(n=2)$; no birth defects were detected in infants. Rates of pregnancy termination in EFV versus NVP users were 34\% versus 23\% ( $P=$ $0.15)$; rates of miscarriage between the two groups were $18 \%$ versus $16 \%(P=0.71)$, respectively.

\section{Discussion}

This is one of the first prospective studies of which we are aware to report pregnancy incidence rates on EFV-based regimens in a clinic setting. Although EFV remains contraindicated during the first trimester of pregnancy in South African guidelines, the pregnancy incidence rate on EFV was very high, 18.6/100 PY. This was observed despite counseling and systematic referrals for regimen changes. Overall, 54 of the 350 women on EFV at enrollment conceived at least once on EFV during study followup. As illustrated in Figure 1, the rate of conceptions on EFV may have been higher in the absence of the study as we routinely screened for fertility intentions and systematically referred women trying to conceive for same day regimen reassessment and change according to the discretion of the facility providers. In the absence of this intervention, the rate of EFV conceptions observed in Figure 1(a) would likely have been even higher and more closely matched incidence rates by participant regimen at the time of study enrollment (Figure 1(b)).

Pregnancy on both EFV and NVP was common in this study, with 170 pregnancies detected amongst 850 women over 12 months of followup. The high rate of pregnancies on EFV resulted from a combination of unplanned pregnancies and planned pregnancies. Fertility intentions and contraceptive use are screened by providers at ART initiation 
TABLE 1: EFV conceptions and regimen substitutions.

\begin{tabular}{|c|c|}
\hline EFV conceptions $(n=56)$ & $n(\%)$ \\
\hline Pregnancies carried forward on EFV & $9(16 \%)$ \\
\hline Women receiving regimen substitutions & $25(45 \%)$ \\
\hline Pregnancies miscarried prior to regimen substitution & $3(5 \%)$ \\
\hline Pregnancies terminated & $19(34 \%)$ \\
\hline Extent of EFV exposure during pregnancy & Median (IQR) \\
\hline Time from EFV conception to detection $(n=56)$, weeks & $4[1-6]$ \\
\hline Time from pregnancy detection to regimen change $(n=25)$, days & $1[1-28]$ \\
\hline Total time of first trimester EFV exposure in pregnancies carried to term $(n=34)$, weeks & $8[5-13]$ \\
\hline \multicolumn{2}{|l|}{ Fertility-related regimen substitutions to NVP or LPV/ $\mathrm{r}^{\dagger}$} \\
\hline Clinical characteristics of women changed from EFV to NVP, $\mathrm{n}=38(67 \%)$ & Median (IQR) \\
\hline $\mathrm{CD} 4$ count, cells $/ \mu \mathrm{L}$ & $257(185-412)$ \\
\hline $\log _{10}$ Viral load, copies/mL & $1.7(1.7-2.1)$ \\
\hline Alanine aminotransferase (ALT) levels, U/L & $26(21-39)$ \\
\hline Clinical characteristics of women changed from EFV to Lopinavir/Ritonavir, $n=19(33 \%)$ & Median (IQR) \\
\hline CD4 count, cells/ $\mu \mathrm{L}$ & $573(333-684)$ \\
\hline $\log _{10}$ Viral load, copies/mL & $1.7(1.7-1.7)$ \\
\hline Alanine aminotransferase (ALT) levels, U/L & $25(19-44)$ \\
\hline
\end{tabular}

${ }^{\dagger}$ Includes regimen changes for EFV conceptions and preventive changes for women trying to conceive.

EFV: Efavirenz; NVP: Nevirapine; LPV/r: Lopinavir/ritonavir.

but are not routinely reassessed over time and may change. This study cohort was a mix of women with ART experience and recently initiating treatment and demonstrates that pregnancy rates on EFV compared to NVP may be lower in the first year after ART initiation, but the overall burden will steadily climb over time (Figure 2). While the overall pregnancy rates reported are higher than those reported by Myer et al. in another South African study [3], pregnancy testing was routinely done at each clinic visit in this cohort and many pregnancies miscarried or terminated would likely have been missed had routine testing not taken place. Amongst women on EFV alone, $50 \%$ of pregnancies were not carried to term. Women on EFV had nonstatistically significantly higher rates of termination of pregnancy than women on NVP, however, the sample size for this analysis was limited. To what extent the terminations were due to concerns over fetal exposure to $\mathrm{EFV}$ is not known; however, counseling was provided to all women to ensure that messages about the low frequency of risk were stressed.

In contrast to guideline recommendations that women on EFV be using a reliable method of contraception, use of hormonal contraception was actually lower amongst women on EFV-based regimens than on NVP. This finding was not expected and further emphasizes the need to reevaluate both fertility intentions and contraceptive use, as method discontinuation amongst women in Sub-Saharan Africa is frequently high [15]. In our data, high rates of EFV conceptions did not appear to be related to reduced efficacy of hormonal contraception amongst EFV users.

The average EFV exposure from conception to regimen change was 6 weeks; this is within the 12-week timeframe recommended for regimen change in the South African treatment guidelines, but beyond what is recommended by the WHO (28 days). Nine women had pregnancies in which the regimen was not changed; the median duration of first trimester EFV exposure amongst all EFV conceptions carried to term was 8 weeks. Regimen substitution after conception is unlikely to happen as quickly in routine clinical practice, as our study tested regularly for pregnancy regardless of last menstrual period or pregnancy symptoms. A South African study of EFV conceptions using pregnancy registry data reported a median gestational age at presentation for regimen change of 19 weeks - well beyond the time of risk for NTD [11]. These results indicate that if regimen changes are delayed amongst women planning conception until pregnancy is detected, fetal exposure to EFV will often be extensive and any potential for harm will likely have already occurred.

We previously reported high rates of fertility intentions at baseline on EFV-based regimens [14]. In the followup data reported here, we also see that many women on EFV are trying to conceive. As noted above, in routine care settings, this will frequently occur as fertility intentions may change after ART initiation, but in the absence of systematic screening, providers may not be aware of these changes. However, our data suggest that routine screening for fertility intentions alone is not sufficient to prevent EFV conceptions, as no consistent approach is being employed to address regimen changes amongst women known to be trying to conceive on $\mathrm{EFV}$. In our study, some women intentionally conceived on EFV despite referrals for regimen changes, as these changes were deferred by providers until pregnancy was established. South African guidelines are clear about what regimens to use at time of initiation if women want children and also specify when to switch regimens in the event of pregnancy. However, the guidelines do not mention how to manage EFV-related fertility concerns amongst stable women trying to conceive before she is pregnant. Clarity is needed 
regarding whether a regimen change should be made for these women and if so, what drugs should be substituted. Inconsistencies in regimen changes may result from lack of specific guidance.

Amongst women receiving regimen changes, either due to EFV conceptions or to prevent EFV conceptions, CD4 count seemed to drive drug substitution choice. Current South African guidelines recommend changing women with EFV conceptions to NVP and do not mention CD4 cell count as a factor that should influence the regimen substitution. Our findings suggest that in practice the guidelines are often not followed, even in sites with relatively strong training programs, and many women appear to have been unnecessarily changed to second-line therapy. This is likely due to hepatotoxicity concerns despite evidence that NVP initiation at higher CD4 counts is safe in women stable on ART [1618].

Given the weak body of evidence surrounding EFV teratogenicity, there is a need to balance a possible risk to the fetus associated with EFV, with the treatment gains that may be achieved amongst adult women. In a recent simulation analysis based on clinical data from Côte d'Ivoire, Ouattara et al. reported that EFV-based regimens would result in substantially fewer deaths at 10 years as compared with NVP use, with only a small increase in birth defects resulting from fetal EFV exposure [19]. The United Kingdom and Malawi have both recently amended ART treatment guidelines to allow for EFV use throughout pregnancy [20, 21]. While it is not the intention of this analysis to advocate for a shift in policy, our findings suggest that there is a need for clarity in the guidelines to foster consistent messages to patients and providers, which will either be intended to reduce rates of conceptions on EFV or to prevent unnecessary regimen changes amongst pregnant women or those trying to conceive while on EFV.

The strength of this study is that it was explicitly designed to compare pregnancy incidence across EFV and NVP users. Pregnancy was routinely and frequently assessed for during followup, and data on fertility intentions and contraceptive use were collected at each study visit. Reasons for changing regimens or continuing on EFV regimens were also collected. The study was not designed, however, to assess EFV teratogenicity; we provide limited data on this; however, the number of live births which were exposed to EFV was too small to provide meaningful information on teratogenicity. Our primary objective was to assess the frequency of pregnancy on EFV and to assess whether guidelines are being followed at the clinic level and whether or not they adequately specify how to address situations experienced in an implementation setting.

\section{Conclusions}

The implications from this study are that high rates of EFV conceptions will take place due to both unplanned pregnancies amongst women not using effective contraception, but also due to planned pregnancies. Delays in providing regimen changes for women trying to conceive on EFV in an environment in which extensive sensitization to possible EFV teratogenicity has previously taken place are notable. For women stable on EFV, clarity over when it is appropriate to substitute regimens and whether women can be changed to NVP at higher CD4 counts should be specified in national and international guidelines. The evidence around EFV teratogenicity is equivocal and many providers may believe that the potential for harm caused to women by changing ART regimens is greater than the risks associated with fetal EFV exposure. However, neither South African nor WHO guidelines reflect this sentiment and consistent guidance around this issue is necessary.

Earlier initiation of ART improves individual survival and has recently been shown to reduce HIV transmission $[22,23]$. Given NVP-related hepatotoxicity concerns, EFV utilization may increase in Sub-Saharan Africa as women initiate ART earlier. Consensus over how to manage EFV use in women with reproductive potential is important in order to minimize either the fetal exposure to EFV or unnecessary regimen changes.

\section{Conflict of Interests}

The authors have no conflicts of interests to declare.

\section{Acknowledgments}

The authors thank the women in the study for their participation, as well as the study staff and sites for their commitment to the participants and the research. The Pregnancy Intentions and Incidence Study received funding from the United States Centers for Disease Control (CDC) public health dissertation Grant 1R36PS001584-01. Sheree Schwartz received support from Grant 1R36PS001584-01, the Department of Epidemiology Doctoral Student Fund at the Johns Hopkins Bloomberg School of Public Health, and the Johns Hopkins Bloomberg School of Public Health Global Field Experience Fund. This research was conducted in clinics supported by PEPFAR and the United States Agency for International Development. All authors contributed to the study design. Sheree Schwartz and Vivan Black oversaw data collection. Sheree Schwartz conducted the analysis with assistance from Shruti Mehta and Taha E. Taha. Sheree Schwartz, Helen Rees, Shruti Mehta, Francis Venter, Taha E. Taha and Vivian Black each contributed substantially to the paper.

\section{References}

[1] R. H. Gray, M. J. Wawer, D. Serwadda et al., "Population-based study of fertility in women with HIV-1 infection in Uganda," Lancet, vol. 351, no. 9096, pp. 98-103, 1998.

[2] J. Homsy, R. Bunnell, D. Moore et al., "Reproductive intentions and outcomes among women on antiretroviral therapy in rural Uganda: a prospective cohort study," PLoS ONE, vol. 4, no. 1, Article ID e4149, 2009.

[3] L. Myer, R. J. Carter, M. Katyal, P. Toro, W. M. El-Sadr, and E. J. Abrams, "Impact of antiretroviral therapy on incidence of pregnancy among HIV-infected women in Sub-Saharan 
Africa: a cohort study," PLoS Medicine, vol. 7, no. 2, Article ID e1000229, 2010.

[4] F. van Leth, P. Phanuphak, K. Ruxrungtham et al., "Comparison of first-line antiretroviral therapy with regimens including nevirapine, efavirenz, or both drugs, plus stavudine and lamivudine: a randomised open-label trial, the 2NN Study," Lancet, vol. 363, no. 9417, pp. 1253-1263, 2004.

[5] SUSTIVA, Important Change in SUSTIVA (efavirenz) Package Insert- Change from Pregnancy Category $C$ to D, SUSTIVA, Washington, DC, USA, 2005.

[6] SUSTIVA, Updated Label Information for Sustiva (efavirenz), SUSTIVA, Washington, DC, USA, 2010.

[7] N. Ford, L. Mofenson, K. Kranzer et al., "Safety of efavirenz in first-trimester of pregnancy: a systematic review and metaanalysis of outcomes from observational cohorts," AIDS, vol. 24, no. 10, pp. 1461-1470, 2010.

[8] World Health Organization, A Systematic Review of the Teratogenicity of Efavirenz, World Health Organization, Geneva, Switzerland, 2009.

[9] N. Ford, A. Calmy, and L. Mofenson, "Safety of efavirenz in the first trimester of pregnancy: an updated systematic review and meta-analysis," AIDS, vol. 25, no. 18, pp. 2301-2304, 2011.

[10] D. K. Ekouevi, P. A. Coffie, E. Ouattara et al., "Pregnancy outcomes in women exposed to efavirenz and nevirapine: an appraisal of the IeDEA West Africa and ANRS databases, abidjan, Côte d'Ivoire," Journal of Acquired Immune Deficiency Syndromes, vol. 56, no. 2, pp. 183-187, 2011.

[11] E. Bera, K. McCausland, R. Nonkwelo, B. Mgudlwa, S. Chacko, and B. Majeke, "Birth defects following exposure to efavirenzbased antiretroviral therapy during pregnancy: a study at a regional south african hospital," AIDS, vol. 24, no. 2, pp. 283$289,2010$.

[12] World Health Organization, Antiretroviral Therapy for HIV Infection in Adults and Adolescents: Recommendations for a Public Health Approach-2010 rev, World Health Organization, Geneva, Switzerland, 2010.

[13] South African Department of Health, Clinical Guidelines for the Management of HIV \& AIDS in Adults and Adolescents, South African Department of Health, Cape Town, South Africa, 2010.

[14] S. R. Schwartz, S. H. Mehta, T. E. Taha, H. V. Rees, F. Venter, and V. Black, "High Pregnancy Intentions and Missed Opportunities for Patient-Provider Communication About Fertility in a South African Cohort of HIV-Positive Women on Antiretroviral Therapy," AIDS and Behavior, pp. 1-10, 2011.

[15] K. Nanda, C. S. Morrison, C. Kwok et al., "Discontinuation of oral contraceptives and depot medroxyprogesterone acetate among women with and without HIV in Uganda, Zimbabwe and Thailand," Contraception, vol. 83, no. 6, pp. 542-548, 2011.

[16] F. van Leth, S. Andrews, B. Grinsztejn et al., "The effect of baseline CD4 cell count and HIV-1 viral load on the efficacy and safety of nevirapine or efavirenz-based first-line HAART," AIDS, vol. 19, no. 5, pp. 463-471, 2005.

[17] E. De Lazzari, A. León, J. A. Arnaiz et al., "Hepatotoxicity of nevirapine in virologically suppressed patients according to gender and CD4 cell counts," HIV Medicine, vol. 9, no. 4, pp. 221-226, 2008.

[18] A. Antela, A. Ocampo, R. Gómez et al., "Liver toxicity after switching or simplifying to nevirapine-based therapy is not related to cd4 cell counts: results of the TOSCANA study," HIV Clinical Trials, vol. 11, no. 1, pp. 11-17, 2010.
[19] E. N. Ouattara, X. Anglaret, A. Y. Wong et al., "Projecting the clinical benefits and risks of using efavirenz-containing antiretroviral therapy regimens in women of childbearing age," AIDS, vol. 26, no. 5, pp. 625-634, 2012.

[20] British HIV Association (BHIVA), Guidelines for the Management of HIV Infection in Pregnant Women 2012. Working Draft, British HIV Association, London, UK, 2012.

[21] E. J. Schouten, A. Jahn, D. Midiani et al., "Prevention of mother-to-child transmission of HIV and the health-related millennium development goals: time for a public health approach," The Lancet, vol. 378, no. 9787, pp. 282-284, 2011.

[22] M. M. Kitahata, S. J. Gange, A. G. Abraham et al., "Effect of early versus deferred antiretroviral therapy for HIV on survival," New England Journal of Medicine, vol. 360, no. 18, pp. 1815-1826, 2009.

[23] M. S. Cohen, Y. Q. Chen, M. McCauley et al., "Prevention of HIV-1 infection with early antiretroviral therapy," New England Journal of Medicine, vol. 365, no. 6, pp. 493-505, 2011. 


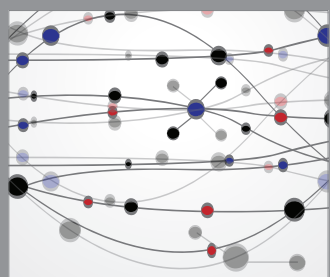

The Scientific World Journal
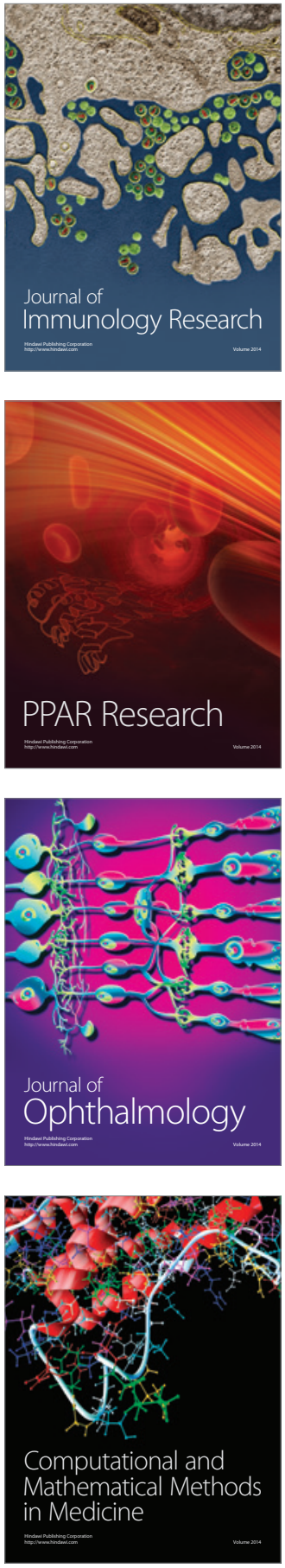

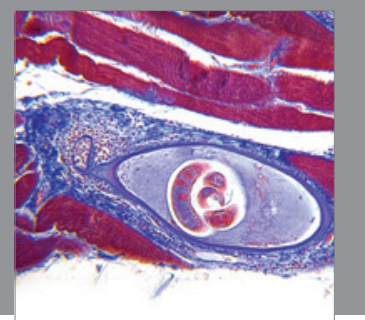

Gastroenterology

Research and Practice
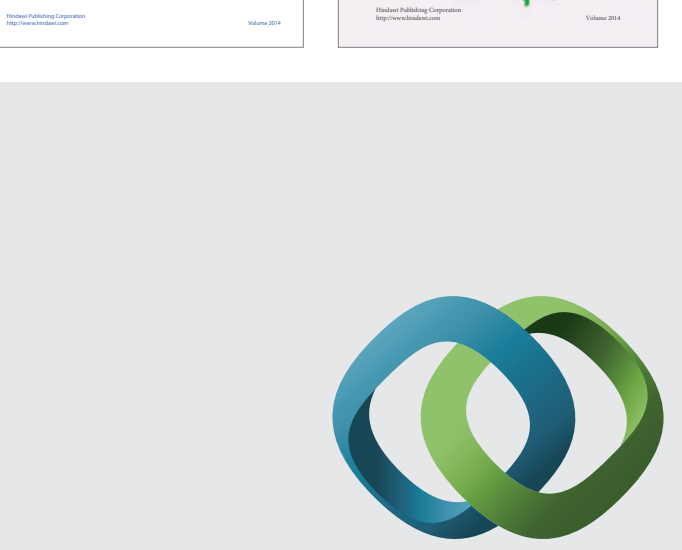

\section{Hindawi}

Submit your manuscripts at

http://www.hindawi.com
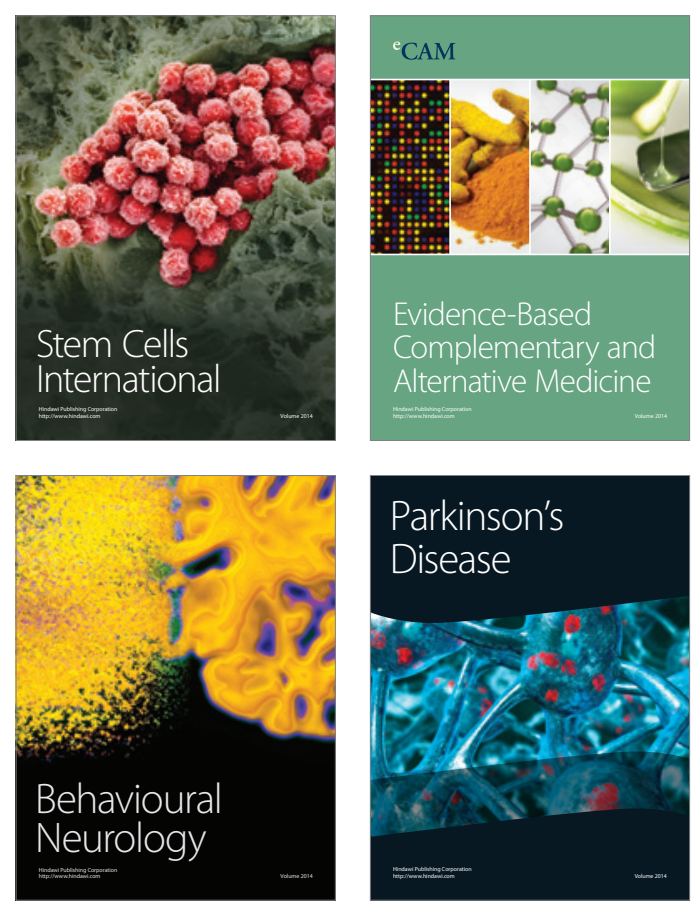

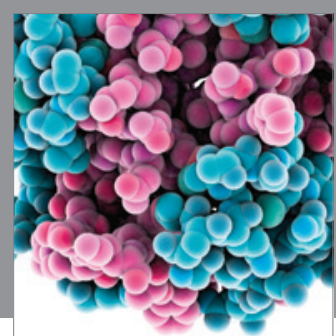

Journal of
Diabetes Research

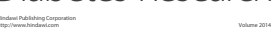

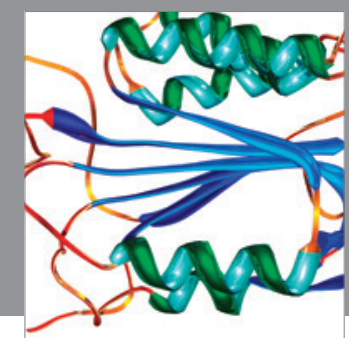

Disease Markers
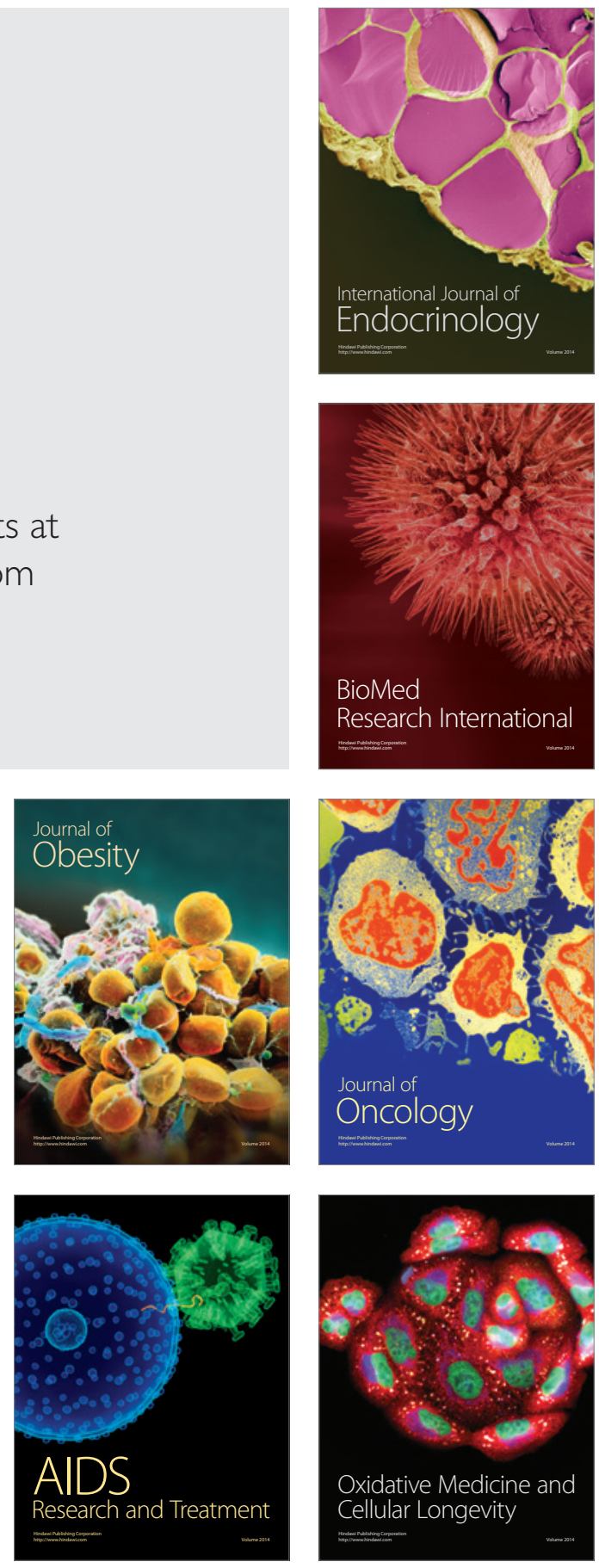